\title{
Paramagnetic Attraction of Impurity-Helium Solids
}

\author{
E.P. Bernard, R.E. Boltnev*, V.V. Khmelenko, \\ and D.M. Lee \\ Laboratory of Atomic and Solid State Physics, Cornell University, \\ Ithaca, NY, 14853 USA \\ * Branch of Institute of Energy Problems of Chemical Physics, \\ Chernogolovka, Moscow region, 142432, Russia
}

\section{INTRODUCTION}

Impurity-helium solids are formed when a mixture of impurity and helium gases enters a volume of superfluid helium. ${ }^{1}$ Typical choices of impurity gas are hydrogen deuteride, deuterium, nitrogen, neon and argon, or a mixture of these. These solids consist of individual impurity atoms and molecules as well as clusters of impurity atoms and molecules covered with layers of solidified helium. The clusters have an imperfect crystalline structure and diameters ranging up to 90 angstroms, depending somewhat on the choice of impurity. Immediately following formation the clusters aggregate into loosely connected porous solids that are submerged in and completely permeated by the liquid helium. ${ }^{2,3}$

Im-He solids are extremely effective at stabilizing high concentrations of free radicals, which can be introduced by applying a high power RF discharge to the impurity gas mixture just before it strikes the superfluid helium. (Figure 1.) Average concentrations of $10^{19}$ nitrogen atoms $/ \mathrm{cm}^{3}$ and $5 \cdot 10^{18}$ deuterium atoms $/ \mathrm{cm}^{3}$ can be achieved this way. Figure 3 shows a typical sample formed from a mixture of atomic and molecular hydrogen and deuterium. Figure 6 shows typical sample formed from atomic and molecular nitrogen. Much of the stability of Im-He solids is attributed to their very large surface area to volume ratio and their permeation by superfluid helium. Heat resulting from a chance meeting and recombination of free radicals is quickly dissipated by the superfluid helium instead of thermally promoting the diffusion of other nearby free radicals. This temperature stability makes Im-He solids an ideal medium in which to study radicals that are involved 


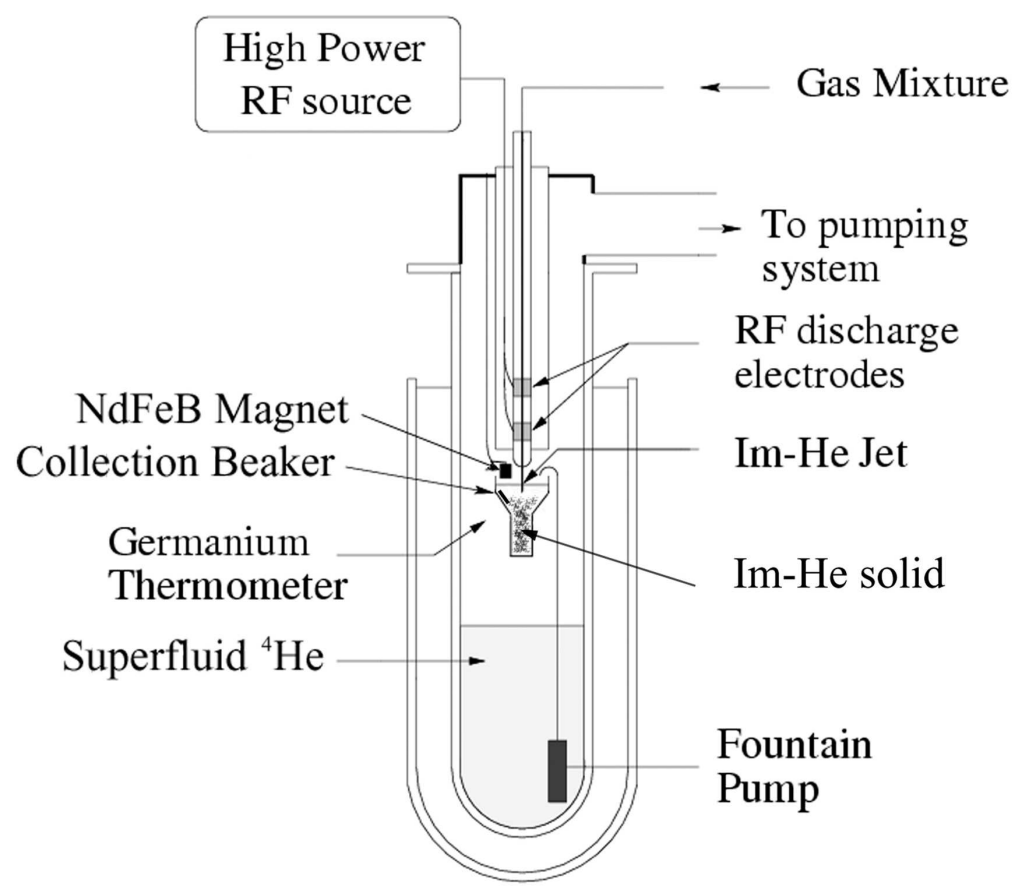

Fig. 1. Experimental apparatus for preparation and paramagnetic lifting of impurity helium solid.

in chemical reactions driven by quantum tunnelling mechanisms as opposed to conventional thermal activation. ${ }^{4}$

\section{Theory of Experiment}

The presence of high concentrations of stabilized radicals in Im-He solids suggests that these samples will have a substantial paramagnetic attraction to regions of high magnetic field. When a permanent magnet is lowered into and withdrawn from a beaker containing an Im-He solid sample, the submerged sample will stick to the magnet if the vertical component of the paramagnetic force exceeds the gravitational force minus the buoyant force provided by the liquid helium that surrounds and permeates the sample:

$$
F_{\mathrm{mag}}=\mu n_{\mathrm{l}} V_{\mathrm{c}} R(\mathbf{B}, T) \nabla \mathbf{B} \cdot \hat{\mathbf{Z}}>\left(\rho_{\mathrm{c}}-\rho_{\mathrm{He}}\right) V_{\mathrm{c}} g
$$

where $F_{\text {mag }}$ is the vertical force exerted by the magnet on a sample, $\mu \simeq$ $\mu_{B} g_{e} J$ is the magnetic moment of each stabilized radical, $V_{\mathrm{c}}$ is the total volume occupied by the impurity clusters in a fragment, $n_{1}$ is the concentration of stabilized free radicals within volume $V_{\mathrm{c}}, \mathbf{B}$ is the field due to the 


\section{Paramagnetic Attraction of Impurity-Helium Solids}

Table 1. Characteristics of gas mixtures and impurity-helium solids.

\begin{tabular}{|l|l|l|c|}
\hline $\begin{array}{l}\text { makeup } \\
\text { gases }\end{array}$ & $\begin{array}{l}\text { respective } \\
\text { admixture }\end{array}$ & $\begin{array}{l}\text { impurities } \\
\text { after discharge }\end{array}$ & $\begin{array}{c}\text { local radical } \\
\text { concentration, } n_{1}\end{array}$ \\
\hline \hline $\mathrm{HD}: \mathrm{D}_{2}: \mathrm{He}$ & $1: 1: 100$ & $\mathrm{H}, \mathrm{D}_{\mathrm{H}}, \mathrm{HD}, \mathrm{D}_{2}$ & $n_{1}>2.5 \cdot 10^{20}$ \\
\hline $\mathrm{D}_{2}: \mathrm{He}$ & $1: 20$ & $\mathrm{D}, \mathrm{D}_{2}$ & $2.9 \cdot 10^{18}<n_{1}<2.5 \cdot 10^{20}$ \\
\hline $\mathrm{N}_{2}: \mathrm{He}$ & $1: 100$ & $\mathrm{~N}, \mathrm{~N}_{2}$ & $8.8 \cdot 10^{18}<n_{1}<8.3 \cdot 10^{19}$ \\
\hline $\mathrm{N}_{2}: \mathrm{He}$ & $1: 200$ & $\mathrm{~N}, \mathrm{~N}_{2}$ & $8.8 \cdot 10^{18}<n_{1}<8.3 \cdot 10^{19}$ \\
\hline
\end{tabular}

permanent magnet, $\hat{\mathbf{Z}}$ is the vertical unit vector, $\rho_{\mathrm{c}}$ and $\rho_{\mathrm{He}}$ are the densities of the impurity clusters and superfluid helium, and $g$ is the acceleration of gravity. The field and temperature dependent Brillouin function ${ }^{5}$

$$
R(\mathbf{B}, T)=\left(1+\frac{1}{2 J}\right) \operatorname{coth}\left[\left(J+\frac{1}{2}\right)\left(\frac{\mu \mathbf{B} \cdot \hat{\mathbf{Z}}}{J k T}\right)\right]-\frac{1}{2 J} \operatorname{coth}\left[\frac{\mu \mathbf{B} \cdot \hat{\mathbf{Z}}}{2 J k T}\right]
$$

accounts for the thermalization of the magnetic moments in the sample, where $J$ is the angular momentum quantum number of the radicals, $k$ is Boltzmann's constant and $T$ is the temperature. Note that $M_{z}=\mu n_{\mathrm{l}} R(\mathbf{B}, T)$ and $M_{z}=\chi \mathbf{B} \cdot \hat{\mathbf{Z}}$ when $\mu \mathbf{B} \cdot \hat{\mathbf{Z}} / k T \ll 1$.

Calculating the force needed to lift samples above the surface of the superfluid helium is complicated by the fact that surface tension causes retention of a substantial amount of liquid helium within the pores of the sample. If the weight and volume of the small amount of helium solidified on the surface of the clusters is ignored, the force needed to withdraw a sample from the helium is:

$$
F_{\mathrm{mag}}=\mu n_{\mathrm{l}} V_{\mathrm{c}} R(\mathbf{B}, T) \nabla \mathbf{B} \cdot \hat{\mathbf{Z}}>\left(\rho_{\mathrm{c}}+\frac{V_{\mathrm{p}}}{V_{\mathrm{c}}} f \rho_{\mathrm{He}}\right) V_{\mathrm{c}} g
$$

where $V_{\mathrm{p}}$ is the volume of the pores in the sample fragment and $f=0.23$ is the degree of saturation of those pores. The porosity $V_{\mathrm{p}} / V_{\mathrm{c}}$ varies greatly from sample to sample, but X-ray studies suggest that $V_{\mathrm{p}} / V_{\mathrm{c}}=150$ is typical of deuterium samples and $V_{\mathrm{p}} / V_{\mathrm{c}}=220$ is typical of nitrogen samples. ${ }^{2,3}$ Measurements of the volume of liquid helium displaced by impurity-helium solids suggest an estimate of $f=0.23{ }^{6}$

\section{RESULTS}

We formed samples at $T=1.5 \mathrm{~K}$ using the four gas mixtures listed in Table 1. Deuterium hydride sample formation and nitrogen sample formation are shown in figures 2 and 5 respectively. Submerged samples within 


\section{E.P. Bernard et al.}

two to three millimeters of the magnet clung to it tightly in each of the experiments. Fragments of submerged deuterium-helium solid and nitrogenhelium solid are shown clinging to the magnet in figures 4 and 7 respectively. At this distance our magnetic field gradient is about $1 \mathrm{~T} / \mathrm{cm}$ and $\mathbf{B} \sim 1 / 2 \mathrm{~T}$. However only the smallest fragments of the $\mathrm{HD}: \mathrm{D}_{2}: \mathrm{He}$ sample stayed in contact with the magnet as it was lifted above the helium surface. If we make the reasonable assumption that our cluster densities are comparable to those of corresponding bulk frozen impurity gases, we find the ranges for $n_{1}$ listed in Table 1.

\section{CONCLUSION}

The experiments show that modest magnetic field gradients $(\sim 1 \mathrm{~T} / \mathrm{cm})$ can exert substantial forces on impurity helium solids. These techniques may be applied in future experiments to move Im-He solids from place to place or to sort them by the concentrations of radicals within their clusters. Refinements of this experiment using magnets specifically shaped to have constant field gradients could greatly improve our crude estimates of $n_{\mathrm{l}}$. To date the formation of pure hydrogen Im-He solids has been hindered by the fact that solid hydrogen floats in superfluid helium. Our group will attempt to form a hydrogen Im-He solid using a magnetic field gradient to counteract the buoyant force and pull the sample into the liquid helium as it is formed.

\section{ACKNOWLEDGMENTS}

This research is supported by NASA grant NAG3-2871 and is based in part on the work of R.E. Boltnev, who was supported by the North Atlantic Treaty Organization under a grant awarded in 2002. We wish to thank Vasiliy Khmelenko for his work on this project.

\section{REFERENCES}

1. E.B. Gordon, V.V. Khmelenko, A.A. Pelmenev, E.A. Popov, and O.F. Pugachev, Chem. Phys. Lett. 155, 301 (1989).

2. S.I. Kiselev, V.V. Khmelenko, D.M. Lee, V. Kiryukhin, R.E. Boltnev, E.B. Gordon, and B. Keimer, Phys. Rev. B 65, 024517 (2002).

3. V.V. Khmelenko, V. Kiryukhin, E.P. Bernard, R.E. Boltnev, S.I. Kiselev, D.M. Lee, (submitted for publication to Phys. Rev. B).

4. S.I. Kiselev, V.V. Khmelenko and D.M. Lee, Phys. Rev. Lett. 89, 175301 (2002). 


\section{Paramagnetic Attraction of Impurity-Helium Solids}

5. F. Reif, Fundamentals of Statistical and Thermal Physics (McGraw-Hill, New York, 1965), p. 259

6. R.E. Boltnev, E.B. Gordon, I.N. Krushinskaya, M.V. Martynenko, A.A. Pelmenev, E.A. Popov, V.V. Khmelenko, A.F. Shestakov, Fiz. Nizk. Temp. 23, 753-756 (1997). [Low Temp. Phys. 23 567-577 (1997).] 


\section{E.P. Bernard et al.}

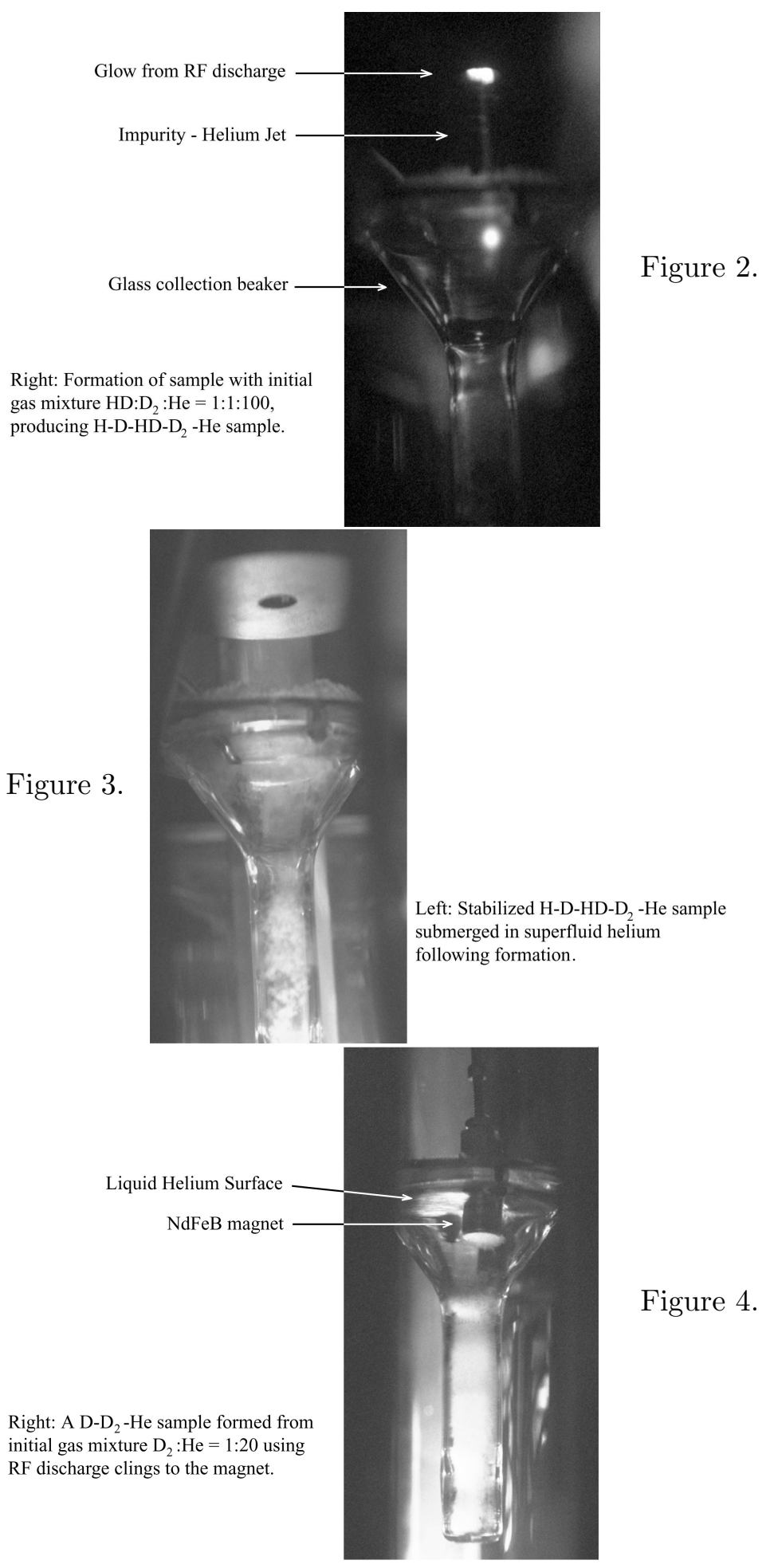


Paramagnetic Attraction of Impurity-Helium Solids

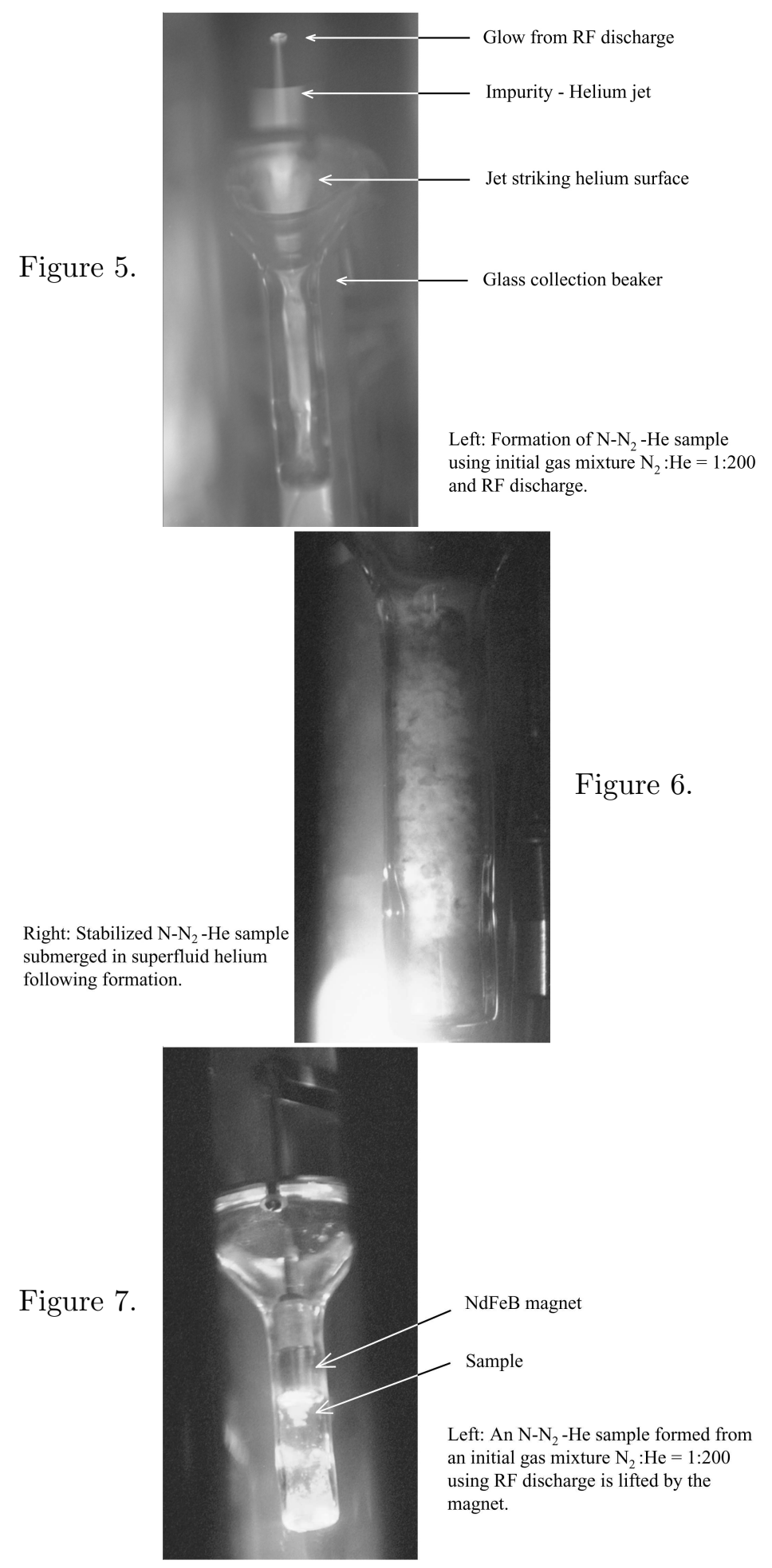

